\title{
472295 - PERFORMANCE PREDICTORS IN ADVANCED CARDIAC LIFE SUPPORT SIMULATIONS
}

\author{
Pamela Morgan, MD, CCFP, FRCPC ${ }^{1}$, Meredith Ford, MSc, MD, FRCPC ${ }^{4}$, Glenn \\ Regehr, $\mathrm{PhD}^{3}$, Michele Haley, BA ${ }^{1}$, Susan DeSousa, BSc, RRT ${ }^{2}$ \\ 1. Anesthesia, Women's College Hospital, Toronto, ON, Canada \\ 2. Anesthesia, Sunnybrook Health Sciences Centre, Toronto, ON, Canada \\ 3. Wilson Centre for Research in Education, Toronto, ON, Canada \\ 4. Anesthesia, University Health Network, Toronto, ON, Canada
}

Introduction: Adherence to Advanced Cardiac Life Support(ACLS) guidelines is generally poor (1-4). Studies have demonstrated that: A-only $9 \%$ of anesthetists adhered to ACLS during a simulated VF arrest(1); B-epinephrine was incorrectly administered during cardiac arrests (2); C-nurses performing BLS protocols did not follow guidelines (3); and D-ACLS not followed in 35.2\% of cardiac arrests (4). The literature suggests that anesthesiologists' adherence to guidelines may be positively affected by recency of ACLS training $(1,6)$ and negatively affected by seniority of the physician (5). High-fidelity simulation (HFS) offers a venue to assess adherence to ACLS guidelines and to explore predictors of ACLS performance.

Methods: After REB approval and consent, practicing anesthesiologists completed a demographic questionnaire including questions about ACLS training (Table). They were randomized to one of 2 HFS scenarios, in which the patient developed pulseless electrical activity (PEA) or ventricular fibrillation (VF). Assessment of performance was conducted using ACLS guidelines and were evaluated in real time by an experienced simulation facilitator. Correlation between questions from the demographic questionnaire and performance was evaluated. Comparisons were made using Pearson correlations with a p value of $\leq 0.05$ considered significant.

Results: Data from 27 anesthesiologists (age 32-64 yrs) were analyzed. There were no statistically significant correlations between adherence to ACLS guidelines and: 1) total number of times taking an ACLS course; 2) recency of ACLS training; 3) total number of codes attended; or 4) number of times being code leader. There were significant negative correlations between adherence to ACLS guidelines and: 1) anesthesiologists' age (rho=0.66, $\mathrm{p}<0.01$ ); and 2) anesthesiologists' years in practice (rho=-0.66, $\mathrm{p}<0.01$ ). Physicians in community practice were less likely to adhere to the guidelines when compared to those from academic centres (rho=-0.38, $\mathrm{p}=0.05$ ).

Discussion: Quiney et al.(5) studied the management of cardiopulmonary resuscitation by anesthetists of varying levels of experience and found that more senior physicians were poorer at resuscitation. Similarly, our study showed a correlation between age, years in practice and ACLS performance. Studies that have shown poor retention of resuscitation skills (6) are also supported by our findings. In a recent review of changing cognitive processing in aging physicians Eva (7) suggested several strategies to improve performance, including providing external supports (e.g. having ACLS algorithms on crash carts), deliberate practice (e.g. regular simulation sessions), as well as education and testing (e.g. updating ACLS certification regularly). 
References: 1) Can J Anesth 1998;45(2):130-132. 2) Acta Anaesthesiol Scand 2004;48:909-913 3) Eur J Emerg Med 2001;8:169-176. 4) Ann Emerg Med 1995;25(1):52-57 5) Resuscitation 1995;29:215-218. 6) Resus 2006;68:101-108 7) Academic Medicine 2002;77(10):S1-S6

\begin{tabular}{|c|c|c|}
\hline Age & Mean \pm SD & $44.81 \pm 6.93$ \\
\hline $\begin{array}{c}\text { Years in Practice } \\
\text { academic centre } \\
\text { community hospital }\end{array}$ & Mean \pm SD & $12.60 \pm 7.84$ \\
\hline $\begin{array}{c}\text { \# ACLS courses taken } \\
\%\end{array}$ & $26.92 \%$ \\
$73.08 \%$
\end{tabular}

